\title{
La pathologie vétérinaire en Guyane française
}

\author{
(Les affections des porcins, des caprins et des ovins)
}

\author{
par H. FLOCH
}

Dans notre étude sur la pathologie vétérinaire en Guyane française, nous avons déjà envisagé les maladies des volailles (1) (2), celles des lapins (3), celles des bovidés (4). Nous allons passer en revue, aujourd'hui, les affections des porcs, des chèvres et des moutons. Elles sont de loin bien moins importantes que celles dont nous avons déjà parlé parce que, d'une part, los élevagos ovins ot caprins sont extrêment réduits en Guyane et que, d'autre part, les porcins sont rarement malades (du moins sous forme d'epizooties) malgré un parasitisme intense, plus particulièrement, nous le verrons, par Stephanurus dentatus et Metastrongylus elongatus.

\section{***}

Sur 87 sérums de porcs guyanais examinés en 1941 , nous en avons trouvé 8 , soit une proportion de $\theta \%$, agglutinant $B$. abortus suis S. 600 à un taux pathologique.

En 1942 et 1943, sur 38 autres sérums de porcs, cinq agglutinaient notre $B$, abortus suis au moins à $1 / 50$.

Sur 159 sérums de porcins guyanais, en 1944 , nous avons trouvé, cette fois, 13 positifs ( $8 \%$ ).

En groupant les résultats de 1945 et ceux deg années précédentes, nous avons finalement les chiffres suivants : 434 séro-diagnostics à $B$. abortus suis pratiqués sur des sérums de porcs guyanais nous ont donné 30 résultats positifs soit la proportion globale de positivité de $9 \%$.

Il nous parait donc indiscutable qu'en Guyane française, à côté de $B$. abortus bovis (4), existe aussi $B$, abortus suis.

D'autre part, il n'est pas sans intérêt de signaler que trois séro-diagnostics pratiqués chez 36 rats gris se sont montrés positifs. Signalons, à ce sujet, que Karkadinonsky aurait pu isoler onze fois sur 36 rats examinés des Brucella: il pense que les rats s'infectent au contact des animaux domestiques, notamment des porcs, et pourraient à leur tour transmettre la maladie.

En 1941, par hémoculture, nous avons pu isoler d'une mélitococcie maligne chez un européen un $B$. melitensis typique (5). Le malade reconnut boire du lait de chèvre cru. Une enquête épidémiologique fut alors effectuée. Il n'a malheureusement pas été possible de déterminer avec certitude où le patient (décédé au vingt-troisième jour de l'évolution de la maladie) s'était infecté. Dix séro-diagnostics ont été pratiqués chez des caprins ( 8 chèvres et 2 boucs) des environs de Susiny où il habitait; un seul bouc a donné une agglutination positive à 1/50. Mais on sait que le séro-diagnostic, épreuve de choix pour les bovidés par exemple, est loin d'avoir la même valeur chez les caprins (il pèche, en général, par défaut). Deux lactoséroagglutinations et dix ophtalmoréactions ont été aussi négatives.

$B$ : melitensis a-t-il été introduit en Guyane en même temps que les chèvres en question au début de cette année I941 ? C'est fort possible car on sait que la plupart du temps l'affection est inapparente chez ces animaux. Mais il est aussi fort possible que cette maladie existait déjà dans le petit troupeau caprin guyanais.

En outre, sur $\theta$ sérums de moutons examinés cette même année 1941, 4 agglutinaient $B$. abortus suis S. 600 à $1 / 50$.

Nous avons déjà dit avoir préparé pour le Service vétérinaire, depuis la guerre, toutes les doses qui lui ont été nécessaires de notre vaccin anticharbonneux «spores-gélose-alun » mis au point en 1942 (4.).

La vaccination du cheptel guyanais a ainsi toujours $\mathrm{pu}$ être effectuée en temps voulu, ce qui n'aurait pu se faire si on avait dû attendre le vaccin d'origine étrangère autrefois seul employé.

Ce dermier vaccin, en granules, ne pouvait servir que pour les bovidés. Notre vaccin-spores, au contraire, peut-être utilisé pour tous les animaux en faisant varier la quantité du produit injecté.

Les injections sont en outre aisées à pratiquer, ce qui est loin d'être négligeable. 


\section{$*^{*} *$}

En 1948, par deux fois, nous avons obtenu, en culture, des Salmonella spp., à partir de foies de porcs.

Le porc est en général, on le sait, considéré comme le plus dangereux des vecteurs de salmoneiles de tous les animaux domestiques; il véhicule fréquemment, en effet, ces germes, dans la zone caraïbe comme ailleurs (6).

Nous avons observé, assez souvent, des cas de tétanos chez des porcs, surtout à la suite de castration.

\section{$* * *$}

Nos examens systématiques sérologiques, pratiqués sur 15 bouchers de Cayenne en 1953, nous ont montré que deux d'entre eux agglutinaient à un taux révélateur Coxiella burneti, l'agent pathogène de la fièvre $Q$.

L'enquête que nous effectuâmes alors nous montra qu'il paraissait vraisemblable que l'un de ces bouchers s'était infecté à l'abattoir de Cayenne en " soufflant » à bouche nue, pour les décoller du tissu sous-cutané, des peaux de chèvres et de moutons, immédiatement après l'abattage; le second s'occupait plutôt de la triperie et de la vente au détail (surtout viande de boeuf, mais aussi viande de porc, de mouton, de chèvre).

Nous avons écrit à ce sujet : "Seule conclusion indiscutable, nos malades n'ayant pas quitté notre département sud-américain, la Guyane fait désormais partie du domaine géographique de la fièvre $Q$ » (7).

Cette rickettsiose se constate surtout chez les personnes en contact direct avec les animaux : vétérinaires, bergers, bouchers, vachers, laitiers, ouvriers travaillant la laine, le crin, les peaux; on l'a signalée dans des maisons situées sur le passage de troupeaux à l'exclusion des autres habitations de la même agglomération.

Chez les ovins et les caprins, l'affection est souvent inapparente, mais on peut y remarquer de la toux (broncho-pneumonie), de l'abattement, de l'anorexie, des avortements, des conjonctivites : C. burneti se trouve dans le lait durant toute la période de lactation chez les animaux infectés.

Chez les bovins la maladie est encore plus inapparente, en général. On décèle C. burneti dans le lait et le placenta des vaches infectées. A l'abattage on peut constater quelquefois une hépatisation pulmonaire, un épanchement pleural, une splénomégalie. Souvent il parait que la glande mammaire, seule, est atteinte. En Oubangui-Chari, les sérums. des bovidés ont été trouvés positifs dans $51 \%$ des cas et dans $81 . \%$ au Ruanda-Urundi.

Les animaux s'infectent vraisemblablement de la même manière que l'homme. Diverses tiques, naturellement infectées par C. burneti jouent sans doute aussi, dans la transmission animale, un rôle réel, mais ce rôle est encore fort mal connu.

$$
* *
$$

Les inspections systématiques des viscères de porcs à l'abattoir nous ont permis de constater le haut degré d'infection de ces animaux par Stephanurus dentatus dont les manifestations se localisent, comme classiquement, au tissu rénal et périrénal. On peut, d'autre part, évaluer à un tiers environ la proportion des foies présentant un ou plusieurs kystes parasitaires à leur surface; ces foies. sont congestionnés, hypertrophiés; parfois l'organe est entièrement envahi de parasites et on y observe des pseudo-tumeurs conjonctives richement infiltrées de polynucléaires éosinophiles.

La stéphanurose porcine est donc très répandue. en Guyane française. Mais comme en d'autre pays on constate que sa gravité n'est en vérité pas très grande dans l'ensemble de l'élevage. Il pourrait en être autrement pour des races améliorées importées. La gravilé de la stéphanurose varie d'ailleurs beaucoup avec les pays; cette affection serait, par exemple, considérée comme bénigne en Annam, mais grave au contraire au Dahomey (8.)

En outre, le parasitisme des poumons de porcs à l'abattoir par un strongle (Metastrongylus elongatus) est si fréquent que l'on pourrait presque condamner systématiquement tous les poumons à Cayenne. La métastrongylose ou broncho-pneumonie vermineuse des porcs est considérée, en général, comme d'un pronostic grave, beaucoup de porcelets mourant de cette affection (8).

A Crique Anguille, en 1943, nous avons constaté la disparition d'un troupeau de porcins provoquée par cette affection; les animaux étaient aussi infectés par Stephanurus dentatus.

A Montjoly, en 1943 encore, des chèvres ont été trouvées parasitées par Esophagostomum venulosum.

\section{***}

Au point de vue parasitisme signalons aussi celui par les puces chiques (Sarcopsylla penetrans) qui décima un troupeau de porcs pendant la guerre, en mars 1952, à Soula, où les porcelets dépérissaient et mouraient, ne pouvant téter à la suite de l'infestation massive des mamelles des truies par les chiques. C'était là, autrefois, un fléau particulièrement répandu dans les communes rurales. Actuellement il a pratiquement disparu grâce à l'emploi du D.D.T., comme nous l'avons souligné ailleurs (9). Institut Pasteur de la Guyane française. 


\section{BIBLIOGRAPHIE}

(1) $\mathrm{FLOCH}(\mathrm{H}$.$) . - Sur la pathologie vétérinaire$ en Guyane française. Les affections des volailles (I), salmonelloses. Archives de I'Institut Pasteur de la Guyane française. Publication no 293, août 1953.

(2) FLOCH (H.). - sur la pathologie vétérinaire en Guyane française. Les affections des volailles (II), pasteurellose, coccidiose, variolo-diphtérie, syngamose, spirochétose, parasitismes divers, tumeurs. Archives de l'Institut Pasteur de la Guyane française. Publication no 295, septembre 1953.

(3) FLOCH (H.). - Les maladies des rongeurs domestiques en Guyane française. Revue d'Elevage et de Médecine vétérinaire des Pays tropicaux, t. VII, no 1, 1954, pp. 5-7.

(4) FLOCH ( $\mathrm{H}:$ ). — La pathologie vétérinaire en Guyane française. Les affections des bovidés. Revue d'Elevage et de Médecine vétérinaire des Pays tropicaux, t. VII, no 3, 1954, pp. 157-163.
(5) FLOCH (H.) et de LAJUDIE (P.). - Etude d'une souche de $B$. melitensis isolée par hémoculture à Cayenne. Archives de l'Institut Pasteur de la Guyane française. Publication no 39 , avril 1942 .

(6) GUILBRIDE (P.-D.-L.). - Veterinary Public Health. Part IV. Fungus Infections, Sylvatic Plague and Salmonellosis. The West Indian Medical Journal. no 2, 1953, p. 439.

(7) FLOCH (H.). - Fièvre Q. Rapport sur le fonctionnement technique de I'I.P. de la Guyane française en 1952. Archives de I'Institut Pasteur de la Guyane française. Publication n'326, mai 1954.

(8) NEVEAU-LEMAIRE (M.). - Traité d'helminthologie médicale et vétérinaire. Paris 1936.

(9) FLOCH (H.). - La quatrième campagne de dédétisation. Ie paludisme en 1951. Archives de I'Institut Pasteur de la Guyane française. Publication no 282, mai 1953. 